\title{
Una propuesta de enseñanza-aprendizaje centrada en el análisis del camino de la energía "paso a paso"
}

\author{
Macarena Soto Alvarado \\ Facultad de Ciencias. Universidad de Santiago de Chile.Chile.macarena.sotoa@usach.cl \\ ORCID: https:/ / orcid.org/0000-0001-8641-4017
}

Digna Couso Lagarón

CRECIM (Centre de Recerca per a l'Educació Cientifica i Matemàtica). Universitat Autònoma de

Barcelona.España.digna.couso@uab.cat

ORCID: bttps:/ / orcid.org/0000-0003-4253-5049

\author{
Víctor López Simó \\ CRECIM (Centre de Recerca per a l'Educació Cientifica i Matemàtica). Universitat Autònoma de \\ Barcelona.España.victor.lopę@uab.cat \\ ORCID: bttps:// orcid.org/0000-0002-2161-9211
}

[Recibido: 12 Abril 2018. Revisado: 1 Octubre 2018. Aceptado: 14 octubre 2018]

\begin{abstract}
Resumen: El modelo escolar de energía y sus ideas clave asociadas (energía como función de estado, transferencia, conservación y degradación) representan un reto para profesores y alumnos, porque requiere cambiar la forma de mirar los fenómenos físicos de la perspectiva mecánica a la energética. Para construir este modelo "paso a paso" presentamos una secuencia didáctica que analiza teórica y experimentalmente un proceso de frenada de una rueda utilizando un sencillo montaje experimental.
\end{abstract}

Palabras clave: Modelización; Transferencia de energía; Degradación de la energía; Educación secundaria.

A teaching-learning proposal focused on the analysis of the energy roadway "step by step"

Abstract: The scholar model of energy and its energy-related concepts (energy as a function of state, transfer, conservation and degradation) represent a challenge for teachers and students, since it implies changing the way to see the physical phenomena, from the mechanics to the energetic view. To build this model "step by step" we present an instructional sequence, analysing both theoretically and experimentally a wheel braking process, using a simple experimental set-up.

Keywords: Modeling; Energy transfer; Energy degradation; Secondary school.

Para citar este artículo: Soto M., Couso D., López V. (2019) Una propuesta centrada en el análisis del camino de la energía "paso a paso". Revista Eureka sobre Enseñanza y Divulgación de las Ciencias 16 (1), 1202. doi: 10.25267/Rev_Eureka_ensen_divulg_cienc.2019.v16.11.1202

\section{Introducción}

La energía no se crea ni se destruye, solo se transforma parece una afirmación científica y profunda que se utiliza a menudo en el aula de ciencias. Desafortunadamente, resulta poco útil para explicar por qué el mundo se está quedando sin recursos energéticos, por qué es saludable desayunar bien para poder hacer actividad durante el día, o por qué unas veces la batería del móvil se descarga más rápido que otras. Construir un modelo escolar de energía en secundaria es un reto difícil, ya que las ideas alternativas de los estudiantes, el lenguaje cotidiano y la propia abstracción del concepto hacen que enseñar energía sea una ardua tarea para el profesorado. Más que memorizar definiciones, aprender sobre energía debería consistir en construir una mirada muy específica a los fenómenos del mundo (de Pro 2015), para comprender y actuar 
mejor ante los importantes retos sociales relacionados con la energía que tiene nuestra sociedad (Solbes y Tarín 2004). En este artículo queremos compartir una reflexión didáctica sobre qué ideas consideramos como claves para un modelo escolar de energía, y mostrar una breve secuencia didáctica especialmente diseñada para construir estas ideas.

\section{Tres ideas clave para construir el modelo escolar de energía}

La discusión sobre qué ideas de energía enseñar en la escuela viene de lejos. ¿Es mejor hablar de transferencia o de transformación? O ¿isi la energía no es una substancia material por qué decimos que se transfiere? No existe una única selección de ideas claves perfecta ni que no acarree algunas contradicciones insalvables. A pesar de ello, creemos que hay que buscar la manera de hablar de energía con nuestros estudiantes que sea lo más coherente con la ciencia y a la vez útil para ellos, cuidando que no obstaculice la comprensión de otros conceptos. Para esto sugerimos usar las siguientes ideas, que surgen de años de discusión en el tema (Ogborn 1986, Pintó 1991, Doménech, Gras-Martí, Guisasola, Martínez-Torregrosa, Salinas, Trumper y Valdés 2003, Millar 2005, López y Pintó 2012a).

IDEA 1. La energía está asociada a la configuración de un sistema, a su estado. Cuando varía el estado de un sistema, varía la energía que le asociamos. La energía no reside en los cuerpos. Es un ente abstracto que asociamos a un cuerpo o sistema en función de cómo está en cada momento y que en primera aproximación podemos relacionar con su potencialidad de producir cambios. Asociamos más energía a un resorte que está comprimido que a uno en su estado natural, o a una taza de agua caliente que a una que está fría. De este modo, podemos concebir el ganar o perder energía con los cambios en el estado (cambios de movimiento, temperatura, compresión, altura, etc.). A través de esta idea, los estudiantes podrán preguntarse cómo estaba cada sistema antes y después, qué cambios se han producido o predecir qué puede suceder y qué no. Esta idea también les permitirá comprender que es imposible medir la energía de un sistema en términos absolutos (Doménech et al. 2003, López y Pintó 2012a) y que aquellos cálculos de energía, que habitualmente realizan en clases de ciencia, corresponden a variaciones en los estados de un sistema. Autores como GarcíaCarmona y Criado (2013) consideran que la idea de energía como propiedad asociada al estado de un sistema es una idea abstracta y que puede generar obstáculos en la comprensión de los procesos energéticos si es trabajada en niveles iniciales, y proponen que sea introducida progresivamente partiendo desde la energía como combustible. Coincidimos en la complejidad de esta idea, así como en la necesidad de su construcción progresiva. Sin embargo, consideramos que al comenzar a trabajar el concepto de energía en base a la observación e interpretación de las variables que cambian en un proceso (es decir, enseñarles a mirar cómo está un sistema antes y después) sirve para construir esta idea abstracta de manera concreta y asequible para el alumnado desde los primeros años. Reducir la idea de energía a la de combustible (aunque sea el punto de partida en los primeros niveles de escolaridad), puede provocar que los estudiantes piensen en la energía en términos de substancia y/o cantidad, así como considerar la energía como un agente causal de los cambios.

IDEA 2. Todo cambio en el estado de un sistema que asociamos a ganar energía lleva asociado otro cambio en el estado de otro sistema a que pierda energía (y viceversa), a lo que le llamamos transferencia de energía. Si la interacción es debida a la diferencia de temperatura entre cuerpos decimos que la energía se transfiere por calor, y si es debida a fuerzas que generan desplazamientos o deformaciones, decimos que se transfiere por trabajo. A pesar que la energía no sea una substancia material, pensar en ella como algo que se transfiere permite seguir las cadenas de cambios sin entrar en los complejos mecanismos que los producen (Millar 2005). Esta perspectiva ayuda a identificar cuáles son los sistemas que experimentan cambios, cuánta energía se ha transferido a cada uno de los 
sistemas que intervienen en un cambio y cómo los cambios en un sistema conllevan cambios en otros. En otras palabras, a través de la idea de transferencia podemos seguir la pista a múltiples procesos entrelazados que forman parte de una cadena energética y comprender la relación entre energía total transferida y energía útil para transferir a nuevos sistemas, además de salir de la lógica de tipos de energía y sus transformaciones, que pueden dificultar una conceptualización correcta del término.

Algunas propuestas didácticas (Pérez-Landazábal y Varela-Nieto 2006, García-Carmona y Criado 2013) consideran fundamental incluir la idea de transformación de energía de un tipo a otro. Si bien no estamos de acuerdo en poner el énfasis en la idea de "transformación", somos conscientes que existen algunos fenómenos en los que se experimentan cambios de estado sin que la energía se transfiera a otro sistema por ejemplo en una caída libre o un lanzamiento parabólico, donde la energía pasa de potencial a cinética o viceversa, y que por lo tanto, en estos casos resulta mucho más intuitivo usar la idea de transformación que la de transferencia.

Por otra parte, los conceptos de calor y trabajo también reflejan dificultades y malos usos, incluso presentes en textos universitarios (Alomá y Malaver 2007), como ser considerados tipos de energía o algo que poseen los cuerpos (Doménech et al. 2003) en vez de ser identificados como mecanismos de transferencia de energía de naturaleza diferente. Es necesario que nuestros estudiantes diferencien estos conceptos e identifiquen que el aprovechamiento de la energía transferida siempre es mayor cuando las transferencias son por trabajo, para poder asociar estas nociones a la idea de eficiencia energética. Por ejemplo, una cocina de inducción siempre será más eficiente que una vitrocerámica de conducción térmica, ya que la primera transfiere energía por trabajo y la segunda por calor.

IDEA 3. La energía puede conservarse en los sistemas aislados, pero siempre se degrada irreversiblemente, perdiendo capacidad para generar nuevos cambios. La idea de conservación de energía, en general, es una idea que se declara sin ser realmente comprendida, y ni mucho menos percibida (Ogborn 1986), ya que en prácticamente todas las situaciones en nuestra vida cotidiana vemos cómo la energía se disipa. Por ello, lo importante es identificar que, en los sistemas no aislados, la energía disipada por un sistema la gana otro sistema o el entorno, de modo que, si se pudieran sumar todas esas energías transferidas entre sistemas, su valor sería el mismo, conservándose. Pero que, a pesar de que se conserve a lo largo de una cadena energética, inevitablemente se irá reduciendo la capacidad que tiene cada sistema para producir nuevos cambios, de modo que a lo largo del proceso la energía cada vez será menos útil y cada vez estará más degradada. La relevancia de esta idea es que puede hacernos pensar en frases que habitualmente escuchamos como consumo de energía o crisis energética, entiendo que la energía no desaparece, sino que se homogeneiza dejando de ser útil.

Somos conscientes del nivel de abstracción de estas ideas, pero entendemos que pueden concretarse de manera comprensible para los alumnos a lo largo de la escolaridad. Por ejemplo, la compleja idea de estado del sistema se puede comenzar a trabajar en primaria simplemente diferenciando cómo son las cosas de cómo están y viendo que en los cambios las cosas pasan a estar a diferente altura, temperatura, movimiento, etc. Así podemos entender que decimos "ganar o perder energía" como una manera de conceptualizar que ha cambiado alguna variable importante, y que nos interesa esa diferencia, por ejemplo aumentar la altura, la temperatura, la compresión, etc. para almacenar energía.

\section{Una propuesta didáctica para construir un modelo de energía}

Para ayudar a los estudiantes a modelizar estas tres ideas clave sobre energía, en el marco del proyecto REVIR se ha diseñado una breve secuencia didáctica para estudiantes de $4^{\circ}$ de ESO, que también puede ser implementada en niveles de Bachillerato, a través de un taller 
experimental de 4 horas, denominado Disipación de la energía por rozamiento (López, Couso y Pintó 2016), el cual sigue un enfoque de enseñanza por indagación basada en modelos (LópezGay, Jiménez Liso y Martínez-Chico 2015). En el taller invitamos a los estudiantes a participar en prácticas de uso y revisión de sus propias ideas iniciales para que estas evolucionen hacia otras más cercanas al modelo escolar de energía; para promover la comprensión del fenómeno, que se ha centrado en el análisis del camino de la energía a lo largo de una cadena de cambios.

El contexto que se presenta a los estudiantes para empezar a discutir sobre energía es un proceso de frenado mecánico de un vehículo. Podemos concebir este proceso desde el momento en que un vehículo está en movimiento (el giro de sus ruedas), posteriormente se frena (que implica no solo el cambio de movimiento, sino también el calentamiento de los frenos por rozamiento), y finalmente el enfriamiento de estos frenos en contacto con el entorno. Hemos seleccionado este fenómeno por varios motivos:

- es sencillo de secuenciar en pasos (primero las ruedas se mueven, luego se paran, etc.)

- ayuda a imaginar las transferencias de energía desde un sistema a otro (de las ruedas a los frenos, de los frenos al aire, etc.)

- obliga a pensar cualitativamente en las ideas de conservación y degradación, superando el enfoque tradicional de análisis cuantitativo de situaciones ideales donde solo actúan fuerzas conservativas.

- es fácil de reproducir en el aula con un montaje experimental sencillo que permita frenar una rueda y medir la temperatura de una lámina metálica situada entre la rueda y las pastillas de freno (figura 1).

Hemos usado una rueda de bicicleta montada en un soporte, la que puede girar libremente y un sistema de frenado hidráulico hecho con una botella hinchable y una lámina de cobre adherida, además de un sensor de temperatura que registra datos a tiempo real (López y Pintó, 2012b). Al hacer girar la rueda de bicicleta y posteriormente presionar la jeringa para frenarla, el sensor mide y grafica el aumento de temperatura de la placa de cobre y el posterior descenso de la temperatura (figura 2). Existen otras maneras de reproducir el fenómeno, usando diferentes tipo de ruedas, sistemas de frenado y sensores de medición.
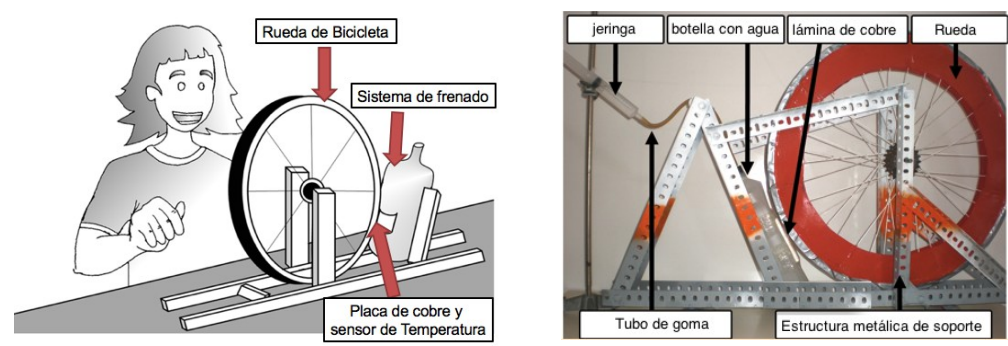

Figura 1. Montaje del sistema de frenado.

Al discutir el origen del aumento inicial de temperatura, este se relaciona con el rozamiento de la rueda con la placa de cobre, es decir, con una transferencia de energía a través de trabajo. De forma análoga, discutimos el descenso de temperatura relacionándolo con una transferencia de energía a través de calor entre la placa a elevada temperatura y el entorno a menos temperatura. Es importante aclarar con los alumnos que el sensor no puede medir ni las transferencia de energía ni el trabajo o el calor, pero sí sus efectos que son el aumento o disminución de temperatura, respectivamente.

Considerando la relevancia de que estudiantes participen en prácticas que realcen la construcción, uso y revisión de modelos para la comprensión de ideas científicas y conocimiento sobre cómo funciona la ciencia (Crujeiras y Jiménez-Aleixandre 2012, Osborne 
2014) es que el diseño de esta actividad contempla que los estudiantes expresen sus ideas, realicen predicciones, evalúen sus respuestas a la luz de nuevas evidencias y revisen sus explicaciones contrastando junto a sus pares para poder construir un modelo científico escolar de energía e interpretar nuevos fenómenos.
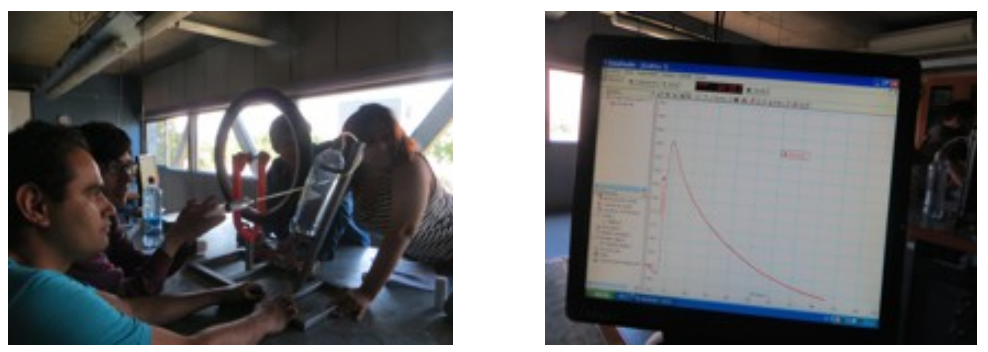

Figura 2. Estudiantes experimentando y gráfica obtenida.

\section{¿Cómo construir la idea de energía como función de estado?}

Para fomentar en los alumnos una mirada "energética" al fenómeno, les proponemos empezar discutiendo cómo está el sistema antes y después de cada uno de los episodios de la cadena. Empezamos centrándonos en los cambios que podemos apreciar y comparar así el "antes vs. después" y les preguntamos explícitamente que señalen "en qué parte del sistema nos fijamos". Esto facilita que expliciten la elección del sistema en el que fijarse y se centren en describir cuál es el estado de cada elemento del sistema en cada momento. Para ello distinguimos diferentes episodios (ver figura 3). Así, en el episodio 1 se dan dos cambios: la rueda pasa de estar en movimiento a estar en reposo y el cobre pasa de estar a temperatura ambiente $\left(T_{\text {amb }}\right)$ a estar a mayor temperatura. Y en el episodio 2 se dan dos cambios más: el cobre pasa de estar caliente a estar a $T_{\text {amb }}$ y el entorno pasa de estar a $T_{\text {amb }}$ a estar ligera e imperceptiblemente calentado. Esto permite centrar la mirada en las variables que cambian y asociar a estas más o menos energía, en lugar de etiquetar los estados con tipos de energía sin saber si alguna variable ha cambiado o no. Es decir, nos permite decir que en el episodio 1 la rueda pierde energía al disminuir su rapidez y la placa aumenta su energía al aumentar su temperatura. Y en el 2, que la placa disminuye su energía al enfriarse y que el aire aumenta su energía al calentarse un poco.
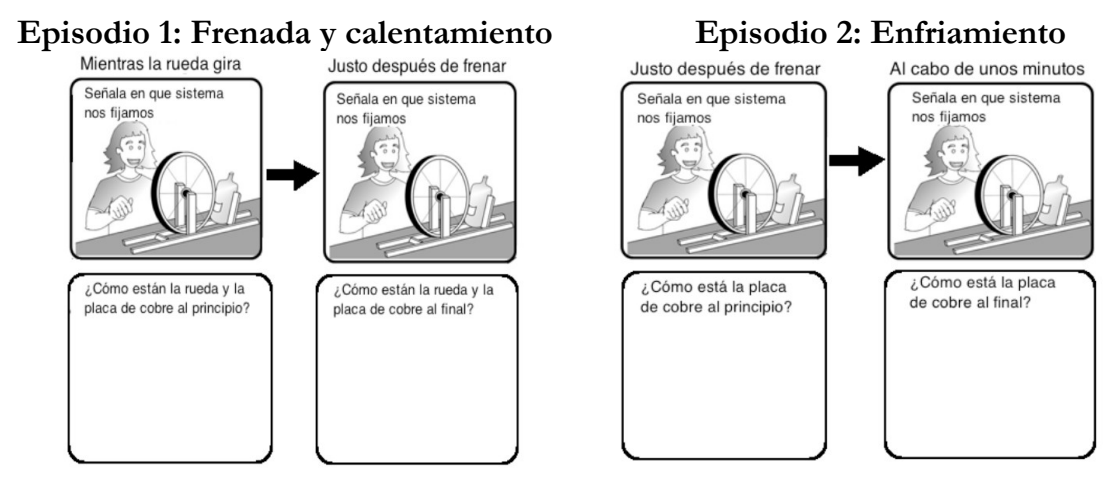

Figura 3. Actividad para promover la idea de estado de sistema.

Esta actividad resulta ser bastante útil para que los estudiantes secuencien el fenómeno e identifiquen las características asociadas a un estado inicial y final. En general, son capaces de identificar que la rueda pasa del movimiento al reposo y la placa de cobre de temperatura ambiente a elevada temperatura (episodio 1), para luego experimentar nuevamente un cambio de una elevada temperatura a una disminución hasta la temperatura ambiente (ver ejemplo en figura 4). Al preguntar en forma directa ¿cómo están la rueda y la placa de cobre al principio/ al final? 
Se pretende que identifiquen las características del estado del sistema, para que posteriormente puedan identificar la interacción, fijándose que en una parte aumentó su energía asociada, mientras que en la otra disminuyó.

\section{Episodio 1: Frenada y calentamiento}

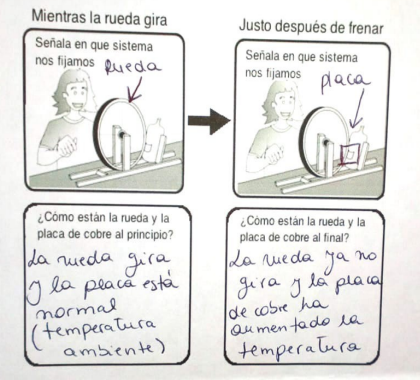

Episodio 2: Enfriamiento

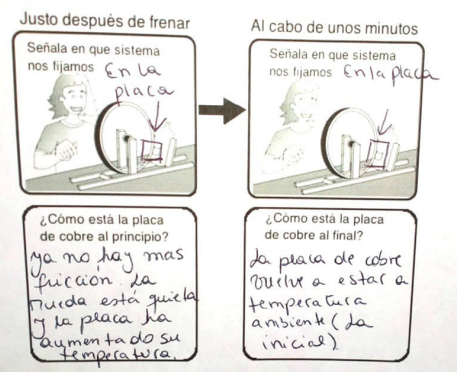

Figura 4. Ejemplo que da un estudiante en la actividad que promueve la idea de estado de sistema.

\section{¿Cómo construir la idea de transferencia de energía a través de calor y trabajo?}

Una vez descritos los estados antes y después de cada episodio, proponemos a los estudiantes poner la mirada en la interacción que causa los dos cambios simultáneos en cada episodio, e identificar qué mecanismo describe mejor cada interacción (ver figura 5). Así, los estudiantes deben señalar a qué parte del sistema asocian la energía en cada etapa, describir los cambios producidos y clasificarlos según si se han producido por la acción de fuerzas (trabajo) o por la interacción de cuerpos a diferente temperatura (calor). A diferencia de lo que suelen creer al principio, se dan cuenta que el cobre se calienta por trabajo (rozamiento), pero se enfría por calor (contacto térmico con el entorno).

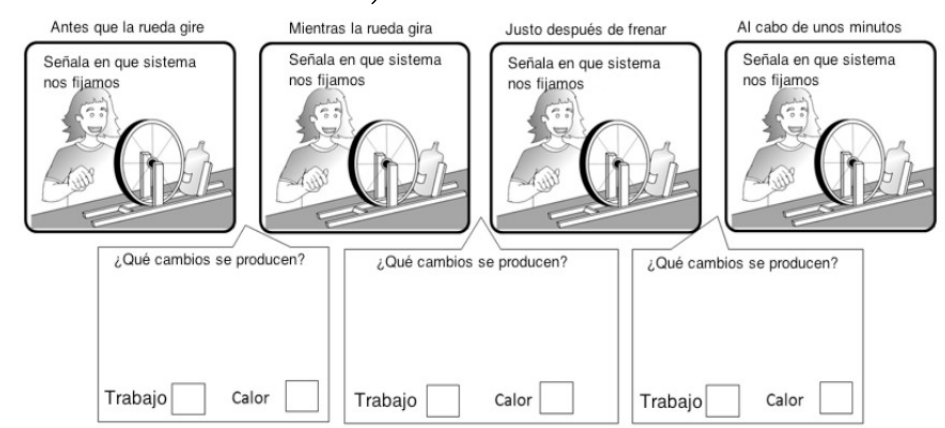

Figura 5. Actividad para promover la idea de transferencia y mecanismos.

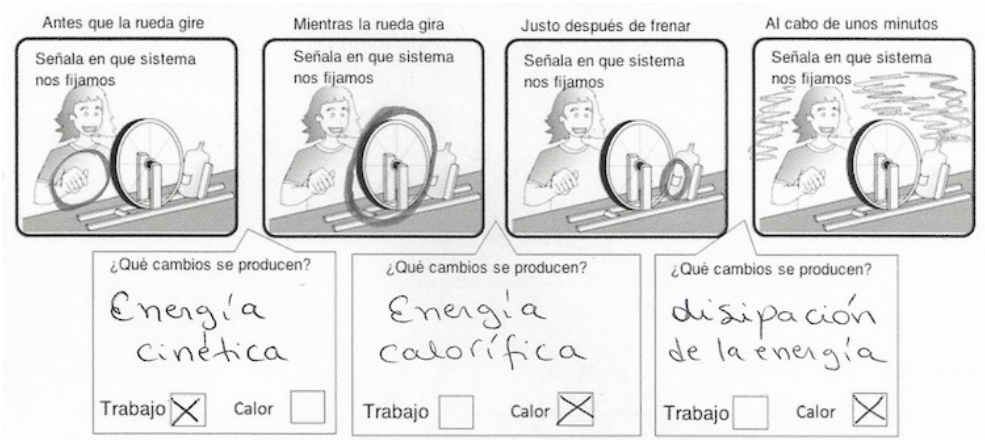

Figura 6. Ejemplo de un estudiante con una lógica de transformación de la energía.

En esta actividad hay estudiantes que responden utilizando un modelo energético cercano al que la actividad pretende potenciar, pero también hay estudiantes que elaboran explicaciones con una visión de transformación de la energía (figura 6). Algunos identifican en forma correcta los mecanismos de transferencia de energía y relacionan los procesos en que 
intervienen fuerzas con la transferencia por trabajo y los procesos de equilibrio térmico con la transferencia por calor (ver figura 7).

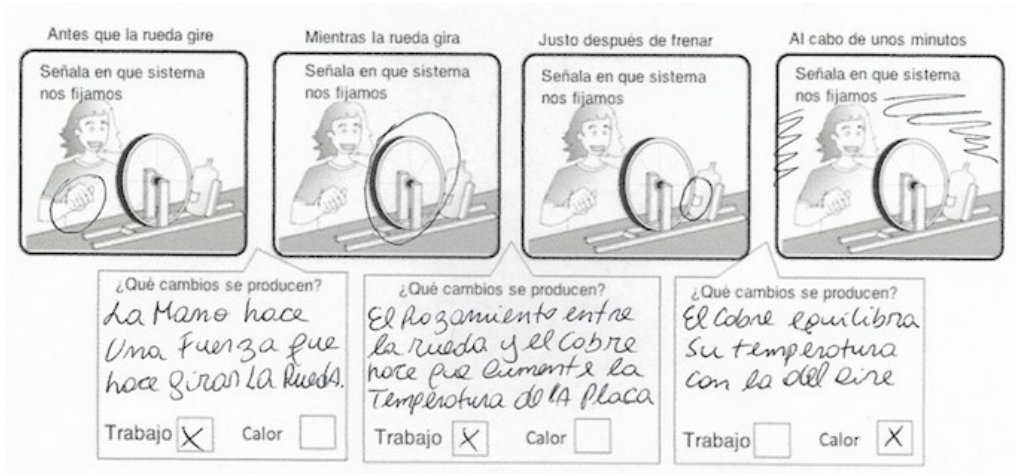

Figura 7. Ejemplo de un estudiante con una lógica de transferencia de energía.

En algunos casos los estudiantes llegan a dar respuestas más completas, dando evidencia de la construcción de la idea de transferencia de energía o incluso incorporando elementos más sofisticados como características microscópicas asociadas al estado del sistema. En la figura 8 se muestra que el estudiante identifica que la placa de cobre se calienta debido a una transferencia de energía a través de trabajo y asocia ese aumento de temperatura a movimiento de las partículas que componen a la placa de cobre.

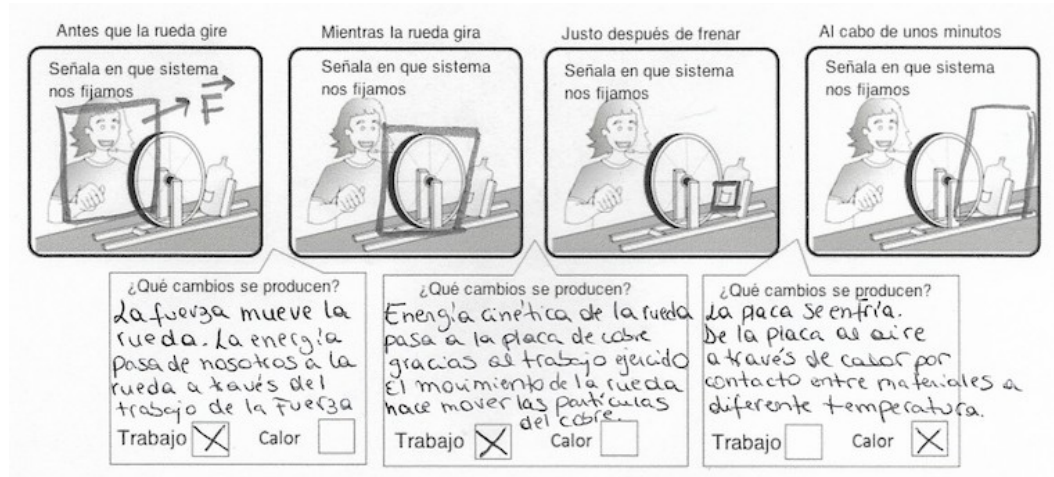

Figura 8. Ejemplo de un estudiante con una lógica de transferencia de energía más sofisticada.

\section{¿Cómo construir la idea de conservación y de degradación?}

Finalmente, se les propone un tercer diagrama con dos nuevas demandas (figura 9). Se les indica "Supongamos que utilizamos $50 \mathrm{~J}$ de energía para mover la rueda con el brazo. Imagínate que podemos seguir el camino de esa energía inicial durante el proceso de frenado. Etiqueta 'cuánta energía' asocias a cada elemento del sistema en cada momento". Esto promueve la discusión entre estudiantes sobre cómo está la energía y cuánta hay en cada momento: si está cada vez más repartida (la energía se disipa en el aire, la rueda, la mesa), y si en todas las viñetas la suma total sigue siendo $50 \mathrm{~J}$. Para profundizar en la idea de conservación en cantidad pero degradación en calidad, también se les pregunta "¿de qué otras maneras se podría aprovechar la energía en cada etapa?”. En la primera viñeta ven que, en vez de hacer girar la rueda, con la misma energía se podría haber lanzado, elevado, etc., provocando así nuevos cambios. En la segunda viñeta, con la rueda ya en movimiento, en vez de frenarla se podría haber hecho mover un generador para encender una luz, o conectarla a una polea para levantar otra masa. En la tercera viñeta, posterior a la frenada y al calentamiento del cobre, las posibilidades de otros cambios imaginables se reducen, pero todavía podríamos usar ese ligero calentamiento para calentar otras cosas. Los estudiantes se dan cuenta en la última viñeta difícilmente hay otros cambios posibles, pues tener el entorno 
ligera e imperceptiblemente a mayor temperatura es un estado energéticamente poco útil, con poca posibilidad de generar nuevos cambios. Esta actividad, lleva a los estudiantes a concluir que el aprovechamiento de energía no es siempre el mismo, que la gama de nuevos cambios posibles se reduce en cada paso, y que en las transferencias por calor la energía es después difícilmente aprovechable para hacer trabajo.

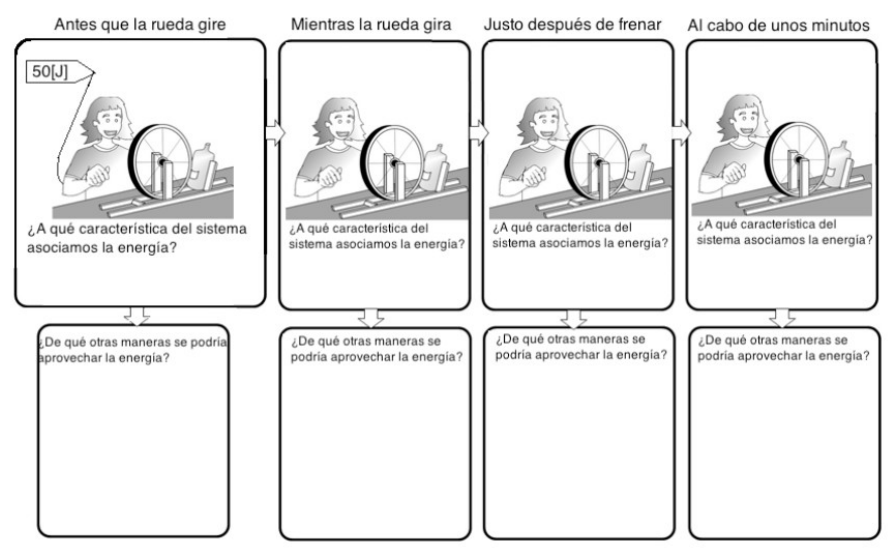

Figura 9. Actividad para promover la idea de conservación y degradación de la energía.

Esta actividad permitió a varios estudiantes ser conscientes de la cadena de cambios, identificando las características del sistema al que se asocia la energía y realizando predicciones de las distribuciones de energía entre las las distintas partes de un sistema (ver figura 10).

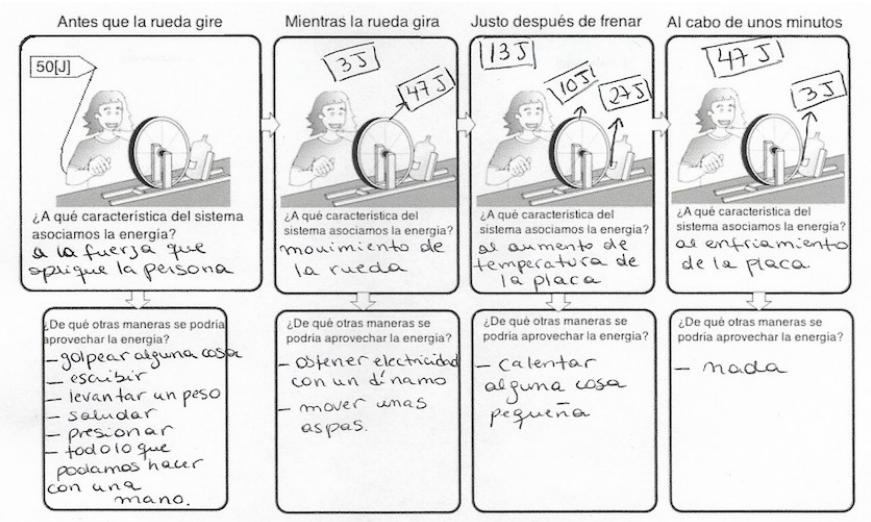

Figura 10. Ejemplo que da un estudiante en la actividad que promueve la idea de conservación y degradación de la energía.

Esta actividad se acompaña por algunas preguntas abiertas (al igual que otras de las actividades ya descritas), con la intención de ir verificando la construcción del modelo de los estudiantes en diferentes momentos del taller. En la siguiente respuesta de un estudiante, posterior a las actividades descritas, se detecta un modelo que incorpora las ideas del modelo científico escolar que proponemos y que mantiene elementos de su modelo mental (tipos de energía): "Partimos del brazo, (energía muy útil) que hace que se mueva la rueda por trabajo, transmitiendo energía cinética, pero una parte se pierde en el ambiente a través de calor. Esta energía (menos útil) a través de la fricción pasa a ser energía calorífica de la placa de cobre, pero pierde una parte más en el ambiente en forma de calor. A medida que la placa de cobre está en contacto con el aire se enfría, transfiriendo calor al ambiente (energía totalmente inútil)".

Al finalizar la secuencia didáctica se propone una aplicación que conecta con el contexto inicial (frenado mecánico de un coche) y que nos permite discutir sobre la idea de ahorro 
energético. En esta actividad los estudiantes, usando las ideas de energía construidas, deben explicar por qué los frenos regenerativos de los coches híbridos o eléctricos son mas eficientes que los de los automóviles convencionales. Esto es debido a que parte de la energía que se transfiere a los frenos no se disipa, sino se reaprovecha para cargar la batería del coche.

\section{A modo de conclusión}

La construcción del camino de la energía que proponemos pretende superar la perspectiva clásica de la enseñanza de la energía centrada en la transformación de diferentes "tipos" de energía, donde una supuesta explicación (que no explica) el fenómeno sería "la energía muscular del brazo se transforma en energía cinética de la rueda, y luego se transforma en energía calorífica en la placa de cobre". Superar esta idea pensando en términos de estado del sistema, de transferencia y de degradación ayuda a los estudiantes a entender estos procesos de forma más competencial, porque les permite actuar. Por ejemplo, que los recursos renovables son cambios que suceden en la naturaleza que podemos aprovechar para generar nuevos cambios útiles para las personas o que en cualquier proceso la eficiencia energética es evitar o minimizar los procesos de degradación.

La implementación de esta secuencia didáctica en un tiempo breve no consigue que los estudiantes construyan todo el modelo científico escolar de energía, ya que para ello se necesitaría un trabajo más extenso y probablemente en otros contextos. Sin embargo, este taller ha sido objeto de análisis didáctico (utilizando para este estudio una comparativa entre dos cursos de $4^{\circ}$ de ESO), y hemos identificado un importante progreso de los modelos de energía que expresan los estudiantes al finalizar del taller en comparación a los que expresaban al principio (Soto, Couso, López y Hernández 2017). Esto nos señala la importancia de futuros trabajos en los que la intervención sea de mayor alcance (una unidad didáctica completa) y se evalúe el uso del modelo en el análisis de nuevos fenómenos.

Las ideas del modelo energético construido pueden ser útiles para el alumnado en otros contextos, como por ejemplo el almacenamiento de energía producido durante la fotosíntesis, la transferencia de energía asociada a la alimentación y a las cadenas tróficas, el aporte calórico de cada tipo de dieta o el movimiento de las placas tectónicas. Es precisamente por el hecho de que el modelo científico escolar de energía se puede aplicar a muchos fenómenos desde distintas disciplinas, que consideramos que dedicar tiempo a trabajar este modelo en el aula vale la pena a pesar de su complejidad.

\section{Referencias}

Alomá E., Malaver M. (2007) Análisis de los conceptos de energía, calor, trabajo y el teorema de Carnot en textos universitarios de termodinámica. Enseñanza de las Ciencias 25 (3), 387-400.

de Pro A. (2015) La energía en la vida cotidiana. Alambique. Didáctica de las Ciencias Experimentales 80, 5-7.

Crujeiras B., Jiménez-Aleixandre M. P. (2012) Participar en las prácticas científicas. Alambique. Didáctica de las Ciencias Experimentales 72, 12-19.

Doménech J. L. L., Gil-Pérez D., Gras-Martí A., Guisasola J., Martínez-Torregrosa J., Salinas J., Trumper R., Valdés P. (2003) Debate para un replanteamiento global. Caderno Brasileiro de Ensino de Física 20 (3), 285-310.

García-Carmona A., Criado A. M. (2013) Enseñanza de la energía en la etapa 6-12 años: un planteamiento desde el ámbito curricular. Enseñanza de las Ciencias 31(3), 87-102. 
López-Gay R., Jiménez Liso M. R., Martínez-Chico M. (2015) Enseñanza de un modelo de energía mediante indagación y uso de sensores. Alambique. Didáctica de las Ciencias Experimentales 80, 38-48.

López V., Couso D., Pintó R. (2016) Dissipació de l'energia per fregament. Seqüència didàctica per a l'estudi de l'energia. Barcelona: CRECIM.

López V., Pintó R. (2012a) Ensenyar energia a secundària. Recursos de Física 9, 1-9.

López V., Pintó R. (2012b) Hot brakes and energy-related concepts: Is energy lost? Physics Education 47 (1), 38-43.

Millar R. (2005) Teacbing about energy Teaching about energy Teacbing about energy. University of York, Department of Educational Studies.

Ogborn J. (2007) Energy and Fuel: The Meaning of "The Go of Things". School Science Review 68 (242), 30-35.

Osborne, J. (2014) Teaching Scientific Practices: Meeting the Challenge of Change. Journal of Science Teacher Education 25 (2), 177-196.

Pérez-Landazábal M. C., Varela-Nieto M. P. (2006) Una propuesta para desarrollar en el alumno de secundaria una visión unificada de la física a partir de la energía. Revista Eureka sobre Enseñanza y Divulgación de las Ciencias 3 (2), 237-250.

Pintó R. (1991) Algunos conceptos implícitos en la $1^{a}$ y la $2^{a}$ Leyes de la Termodinámica: una aportación al estudio de las dificultades de su aprendizaje. Tesis Doctoral. Universitat Autònoma de Barcelona.

Solbes J., Tarín F. (2004) La conservación de la energía: un principio de toda la física. Una propuesta y unos resultados. Enseñanza de las Ciencias 20 (3), 415-425.

Soto M., Couso D., López V., Hernández M. I. (2017) Promoviendo la apropiación del modelo de energía en estudiantes de $4^{\circ}$ de ESO a través del diseño didáctico. Ápice. Revista de Educación Científica 1 (1), 90-106. 